\title{
Editorial
}

\section{Hypertension in the Time of the COVID-19 Pandemic: New Issues and Enduring Controversies}

\author{
Suzanne Oparil, $M D$ \\ Vascular Biology and Hypertension Program, Division of Cardiovascular Disease, Department of Medicine, University of \\ Alabama at Birmingham, Birmingham, Alabama
}

In his recent review, "Future of Hypertension: The Need for Transformation," Victor Dzau, president of the National Academy of Medicine, emphasized the need for sweeping transformation in the ways we diagnose and treat hypertension so as to reduce the global burden of the disease (1). The review emphasizes the role of emerging technologies in changing the way healthcare is delivered, with an overall trend shifting away from hospitals and clinics toward care delivered on a daily basis in the home. This will require improved technologies for out-of-office BP monitoring, along with enhanced digital technologies for the transfer and analysis of BP information so as to identify and deliver optimal treatment regimens that are evidence based (Figure 1). The five key areas in which progress is needed to advance hypertension treatment and control are depicted in this figure.

The personal isolation and distancing strategies mandated in the COVID-19 pandemic accelerate the need for telemedicine approaches for monitoring home BPs and delivering the values to the healthcare provider in a manner that can be readily applied to managing antihypertensive medical regimens. A variety of devices for noninvasive continuous BP monitoring are currently available $(2,3)$, and more are on the way (4). These devices have their greatest potential to revolutionize the care of the hypertensive patient when coupled with artificial intelligence, the science and engineering of making intelligent machines (computer programs), in the healthcare system (5). Artificial intelligence, coupled with accurate monitoring devices, can improve access to care and provide more detailed and accurate BP monitoring, thus enhancing clinical decision making. Furthermore, increased patient engagement in these processes may enhance adherence to prescribed medical and lifestyle regimens, thus slowing the progression of hypertension and preventing its cardiovascular disease (CVD) complications. In addition to the need for improved diagnosis and treatment of hypertension in the out-ofoffice setting, there is continuing need for fundamental research on the pathogenesis of hypertension and its cardiovascular and renal complications, with the ultimate goal of developing novel targeted therapies that are well tolerated and effective in not only lowering BP but also preventing target organ damage.

The National Heart, Lung and Blood Institute (NHLBI) recently weighed in on the issue of translating basic research to improve control of hypertension (6). The NHLBI Working Group on Hypertension reviewed recent discoveries that may be ready for testing in clinical trials and highlighted the most promising areas in which to improve translation. It also identified gaps in knowledge that appear to impede translation, and key challenges and barriers to moving basic science discoveries into the clinic. Finally, the Working Group identified several research opportunities that promise to enhance the translation of hypertension research to clinical care (Table 1). Overall, the Working Group concluded that multilevel multicomponent strategies, along with patient-level strategies, are needed to improve BP control in hypertensive patients. Nonadherence to prescribed medical regimens and clinical inertia were found to be major barriers to BP control. The panel concluded that enhancements in the field of implementation science are needed to ensure that successful programs of BP control reach the large and growing population of hypertensive patients in a timely manner.

Currently, several controversies in hypertension management deserve particular attention.

\section{Renin-Angiotensin-Aldosterone System Inhibitor Use During the COVID-19 Pandemic}

Angiotensin-converting enzyme 2 (ACE2), the monocarboxypeptidase that inactivates angiotensin II and thereby counters the activation of the classical renin-angiotensin-aldosterone system (RAAS), has been identified as the functional receptor for the severe acute respiratory syndrome coronavirus 2 (SARS-CoV-2) (Figure 2) (7). The SARS$\mathrm{CoV}-2$ virus is responsible for the COVID-19 pandemic (8). The virus has spike proteins that bind to ACE2 on the surface of lung alveolar epithelial cells. The virus is then endocytosed, causing downregulation of surface ACE2 and accumulation of angiotensin II in the lung, with attendant increases in oxidative injury. The coronavirus replicates in the lung, further damaging lung tissue.

Early reports of clinical characteristics of patients with COVID-19 in China found a high prevalence of hypertension, CVD, and diabetes in those with more severe forms of the disease $(9,10)$. These comorbidities are commonly treated with ACE inhibitors or angiotensin receptor blockers (ARBs). ACE inhibitors and ARBs have been shown to upregulate ACE2 in some animal models $(11,12)$ and in some patients with hypertension or diabetes (13). These observations have raised concerns that the ACE inhibitors or ARBs may increase expression of ACE2, the known receptor for coronavirus, and thus increase the risk of infection with COVID-19 or worsen the severity of COVID-19 in those who are already infected (14). These issues have led to extensive discussion in the literature and in medical societies around the world about three critical questions that are of great importance to patients and healthcare providers in the setting of the COVID-19 pandemic $(15,16)$ : 1) Is there a link between hypertension and increased risk of COVID-19 infection in humans? 2) Are patients on ACE inhibitors/ARBs at an increased risk of infection or severity of disease? 3) Does starting, stopping, or continuing these drugs lead to better or worse outcomes in COVID-19?

Whereas hypertension has been reported as the most prevalent coexisting condition in patients with severe COVID-19 in China, 


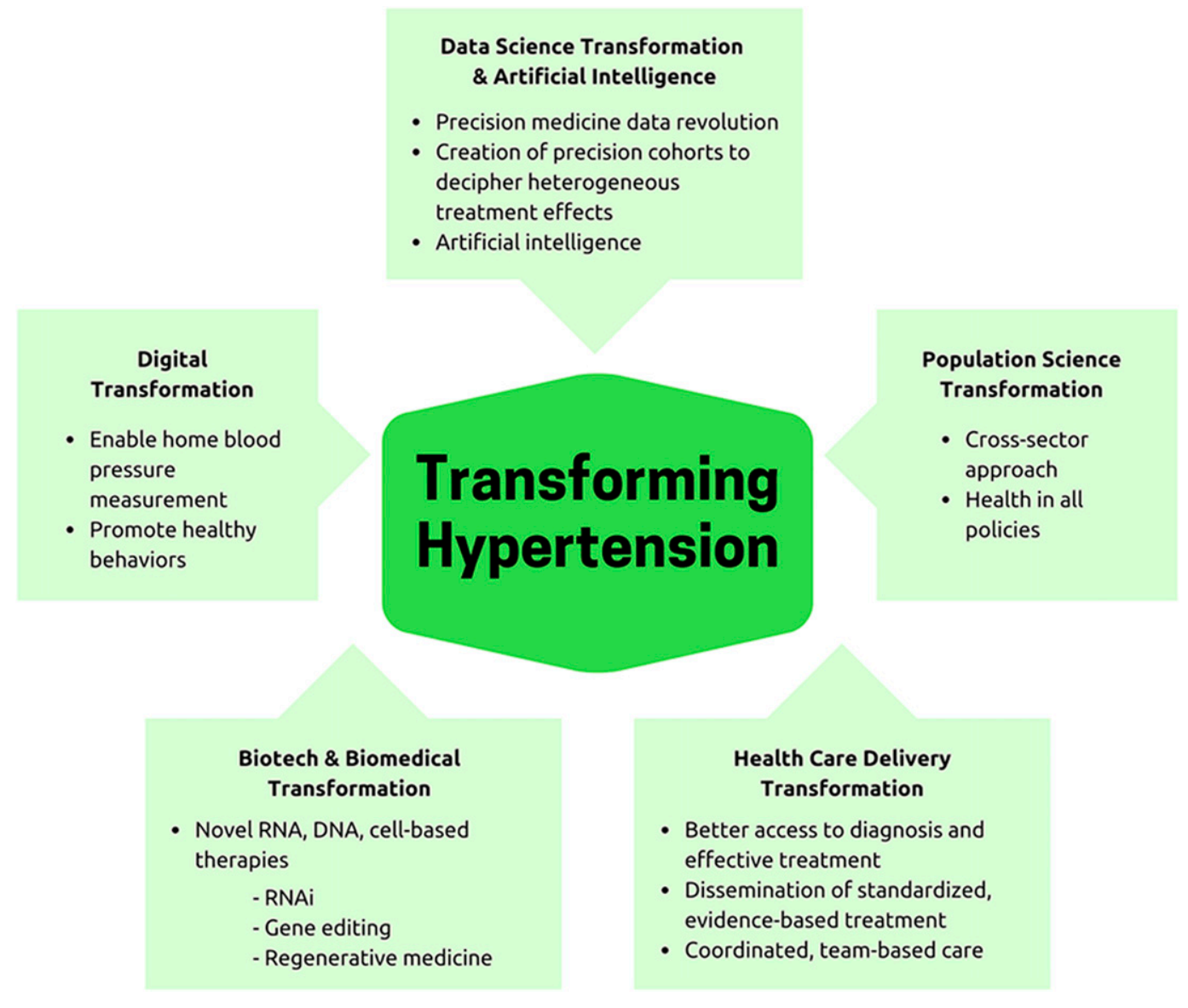

Figure 1. Hypertension: need for transformation. To control or eliminate hypertension, there is a need for system-wide transformation in research and clinical care and in the convergence of disciplines. This figure highlights the five key areas where progress is needed to advance hypertension control and treatment. Achieving maximum benefit will require convergence of these areas. Used with permission from Figure 1 of reference 1 (Dzau VJ, Balatbat CA: Future of hypertension. Hypertension 74: 450-457, 2019).

this relationship is confounded by the strong association with advancing age, which is currently the strongest predictor of COVID19-related death (17). Furthermore, data on ACE inhibitor/ARB use in hypertensive Chinese patients before and during the course of COVID-19 are sparse. Further study is needed to determine whether hypertensive persons are at increased risk of COVID-19 infection/ severity of disease.

Data on the effects of ACE inhibitors and ARBs on ACE2 levels or activity in animal models are inconsistent, with some studies showing increases in ACE2 mRNA or protein levels and others showing no effect $(11,12,18,19)$. Furthermore, data on the effects of ACE inhibitors and ARBs on ACE2 expression and ACE2-directed angiotensin II metabolism in humans are sparse and inconsistent, and data on the effects of RAAS inhibitors on lung-specific expression of ACE2 are nonexistent (8). Contrary to the hypothesis that RAAS blockers predispose to lung injury in the setting of COVID-19 infection, there is evidence that ACE inhibitors and ARBs can lessen the extent of coronavirus-mediated acute lung injury by maintaining ACE2 levels and thus reducing generation of angiotensin II in lung (20). Similarly, administration of recombinant ACE2 protein has been shown to protect against lung injury induced by respiratory syncytial virus injection in animal models (21) and to reduce angiotensin II levels in a phase 2 trial in humans with acute respiratory distress syndrome (22). Consistent with these preliminary observations, clinical trials of recombinant ACE2 protein and of the ARB losartan are under way in patients with COVID-19 to restore balance to the RAAS and prevent organ injury (8).

In contrast to the uncertainty about the potential benefit of initiating RAAS blocker use in patients with COVID-19, there is a clear potential for harm in withdrawing these agents in high-risk COVID-19 patients with established myocardial injury, hypertension, or heart failure (8). Abrupt withdrawal of RAAS blockers from clinically stable patients with COVID-19 and these comorbidities may result in clinical instability and adverse health outcomes. Accordingly, major medical societies recommend continuing ACE inhibitors and ARBs in hypertensive patients with COVID-19 because of lack of evidence of benefit and strong evidence of harm from discontinuation (Table 2). Some specifically conclude that routine use of ACE inhibitors or ARBs to treat hypertension should not be influenced by concerns about COVID-19 in the absence of compelling data that ACE inhibitors/ARBs either improve or worsen susceptibility to COVID-19 infection or affect the outcomes in those infected (23). These recommendations apply to patients who are in stable condition and are at risk for, being evaluated for, or infected with COVID-19 (8). 


\section{Table 1. Research opportunities to enhance translation of hypertension research}

Encourage innovative translational research that requires collaboration among basic and clinical scientists and includes patient-oriented research.

Facilitate training that encourages collaboration and cross-training in basic science and clinical application.

Develop new drugs and treatments (such as potassium-rich diets) to target diverse hypertensive patient populations, such as patients with resistant hypertension.

Capitalize on resources currently or previously supported by NHLBI, such as databases, clinical populations, and clinical trial data, that will facilitate discovery.

Develop new technologies for better phenotyping of humans and animals through in vivo imaging, single-cell analysis (central repository and analysis), analysis of large data sets, validation of surrogate endpoints and biomarkers, robust long-term follow-up, and assessment of tissueand organ-based sympathetic activity.

Support studies on hypertension and aging, including arterial aging, cognition, medication adherence, and complications of antihypertensive therapy.

Support studies related to the role of sex differences in the complications of hypertension and hypertension in pregnancy and preeclampsia.

Develop and use animal models that are best suited to the scientific question posed irrespective of cost.

Develop approaches to optimally detect and reverse antihypertensive medication nonadherence.

Strengthen the evidence base for genetic screening tools for both risk of hypertension and optimal treatment options, with collection of genetic data in clinical trials and population-based studies across the lifespan.

Support clinical trials for early intervention in high BP, particularly in stage 1 hypertension and in younger populations, with long-term tracking of outcomes.

Develop strategies to engage healthcare practitioners in strong patient relationship bonds and trust to promote lifestyle modification in high-risk populations.

Support studies that focus on multilevel, collaborative system-based approaches, including patients, providers, and health systems (at a minimum of 2 levels).

Encourage researchers to incorporate implementation science methodologies that can look broadly to bridge healthcare and community settings.

Support clinical trials designed to use quasi-experimental or mixed methodologies and those that specifically address the questions, such as "who does it work for?" and "when does it work?"

Convene representatives and leaders from NHLBI, healthcare systems, payers, industry, insurers, and other government agencies to address implementation science in hypertension.

Support training for the next generation of health disparities and implementation science researchers, including laypersons and community health workers.

Used with permission from Table 2 of reference 6 (Sigmund CD, Carey RM, Appel LJ, et al.: Report of the National Heart, Lung, and Blood Institute Working Group on Hypertension: barriers to translation. Hypertension 75: 902-917, 2020).

NHLBI, National Heart, Lung, and Blood Institute.

Further research that will address key unanswered questions about the role of the RAAS in the pathogenesis and possible treatment of COVID-19 and other coronavirus-based diseases is urgently needed.

\section{Timing of Antihypertensive Medication Dosing}

Awareness of diurnal rhythms in BP, and particularly the importance of nocturnal BP in determining risk of CVD, has led to discussions about the appropriate timing of antihypertensive medication dosing. Ambulatory BP monitoring studies have shown that nighttime BP levels are more predictive of CVD outcomes than 24-hour or daytime levels and that dipping status also may be an independent predictor of outcomes (24). A series of studies, including the Prognostic Value of Ambulatory Blood Pressure Monitoring in the Prediction of
Cardiovascular Events and Effects of Chronotherapy in Relation to Risk (MAPEC) study (25) and the Heart Outcomes Prevention Evaluation (HOPE) study (26), culminating in the recently published Hygia Chronotherapy Trial (27), have reported beneficial effects of bedtime hypertension treatment on CVD risk. The MAPEC study enrolled 2156 patients with uncontrolled BP on three antihypertensive agents taken in the morning and randomized them to either continue their current regimen or take one of the medications in the evening and two in the morning. MAPEC reported that administration of at least one antihypertensive agent at bedtime, compared with morning-only dosing, was associated with better BP control overall and with reduction in nondipping BP patterns and CVD events. The HOPE trial showed that, compared with placebo 


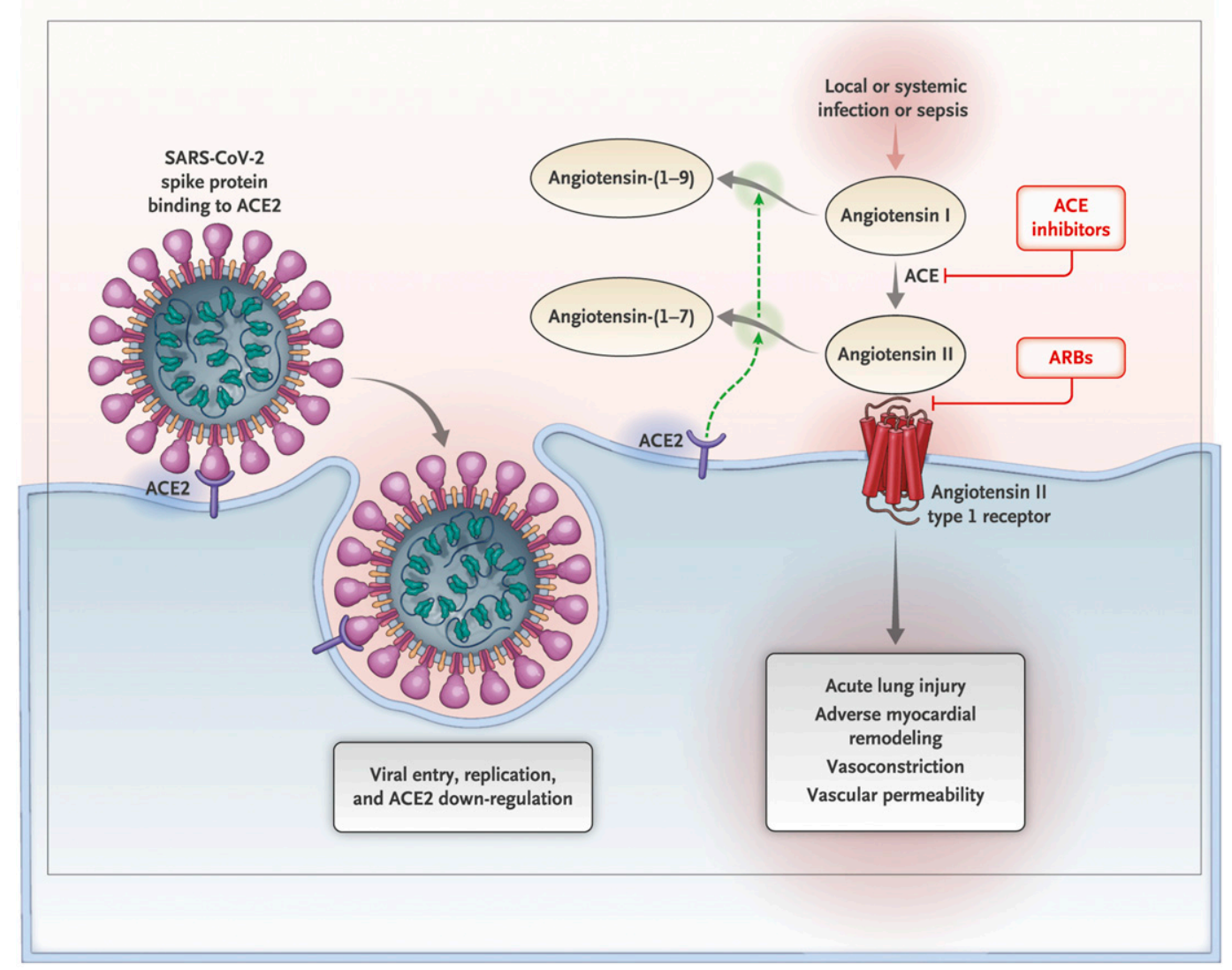

Figure 2. Interaction between SARS-CoV-2 and the renin-angiotensin-aldosterone system. Shown is the initial entry of severe acute respiratory syndrome coronavirus 2 (SARS-CoV-2) into cells, primarily type II pneumocytes, after binding to its functional receptor, angiotensinconverting enzyme 2 (ACE2). After endocytosis of the viral complex, surface ACE2 is further downregulated, resulting in unopposed angiotensin II accumulation. Local activation of the renin-angiotensin-aldosterone system may mediate lung injury responses to viral insults. ACE, angiotensin-converting enzyme; ARB, angiotensin receptor blocker. Reprinted with permission from reference 8 (Vaduganathan M, Vardeny O, Michel T, McMurray J, Pfeffer MA, Solomon SD: Renin-angiotensin-aldosterone system inhibitors in patients with Covid-19. N Engl J Med 382: 1653-1659, 2020). Copyright (C) (2020) Massachusetts Medical Society. Reprinted with permission from Massachusetts Medical Society.

control, nighttime dosing with ramipril resulted in large reductions in CVD outcomes without major effects on daytime office BP.

The Hygia Chronotherapy Trial enrolled 19,084 hypertensive patients who underwent 48-hour ambulatory BP monitoring at baseline and at yearly intervals throughout the study (median follow-up time 6.3 years). At the end of the study, nighttime ambulatory BP was significantly lower $(114.7 / 64.5 \mathrm{mmHg})$ in the bedtime-dosing group than in the morning-dosing group $(118.0 / 66.1 \mathrm{mmHg})$, and daytime ambulatory BP was nearly identical in the two groups. The mean number of antihypertensive drugs taken at the end of the study was significantly lower (1.71) in the bedtime group compared with the morning group $(1.80, P<0.001)$. Dramatic reductions were reported in all-cause mortality and cardiovascular mortality and in CVD-outcome events, including myocardial infarction, heart failure, stroke, and coronary revascularization, with adjusted hazard ratios for total events $=$ 0.58 and for all-cause mortality $=0.55$.

The report of the Hygia study (27) and the accompanying editorial (28) have been widely discussed among hypertension researchers and clinicians, in the lay press, and among patients. Major concerns have been expressed related to study design (uncertainty whether it was really a randomized controlled trial that conformed to common standards ([CONSORT]), statistical issues, and questions about adequacy of monitoring and the independence of adjudicators of study outcomes (29-31). Most importantly, critics have questioned how such dramatic reductions in mortality and CVD events could have been related to the very modest between-group differences in BP that were reported.

Earlier studies have shown that excessive nighttime lowering of $\mathrm{BP}$, including overdipping or extreme dipping, is associated with increased risk of ischemic injury in patients with coronary artery disease (32) or silent cerebral infarcts (33), particularly in the elderly (34). These findings suggest that bedtime dosing of antihypertensive medications could harm those patients by further reducing their nighttime BPs. The recommendation for bedtime dosing of BPlowering medications has also been questioned based on evidence that adherence to prescribed antihypertensive medications, documented by using the Medication Event Monitoring System (MEMS), is significantly lower when medications are taken at bedtime versus morning (35)

Numerous well-designed randomized controlled trials of hypertension treatment, with hundreds of thousands of participants, have dosed the medication in the morning rather than at bedtime. 
Table 2. Professional societies' recommendations for use of ACE inhibitors/ARBs in COVID-19 patients

Society

European Society of Hypertension

European Society of Cardiology

Council on Hypertension

Hypertension Canada

Canadian Cardiovascular Society

The Renal Association, United

Kingdom

International Society of Hypertension

American College of Physicians

Spanish Society of Hypertension

American Heart Association, Heart

Failure Society of America,

American College of Cardiology

European Renal Association -

European Dialysis and Transplant

Association

American Society of Pediatric

Nephrology

High Blood Pressure Research

Council of Australia

Australian Diabetes Society
Summary of Recommendations

Last Statement Update

Recommend continuing ACEs/ARBs due to lack of evidence to support differential use in COVID-19 patients. In those with severe symptoms of sepsis, antihypertensive decisions should be made on a case-by-case basis taking into account current guidelines

Strongly encourage continuing ACEs/ARBs due to lack of evidence to support discontinuing

Recommend continuing ACEs/ARBs due to lack of evidence that patients with hypertension or those treated with ACEs/ARBs are at higher risk of adverse outcomes from COVID-19 infection

Strongly encourage continuing ACEs/ARBs and Angiotensin Receptor Neprilysin Inhibitors due to a lack of clinical evidence to support withdrawal of these agents

Strongly encourage continuing ACEs/ARBs due to unconvincing evidence that these medications increase risk

Strongly recommend that the routine use of ACEs/ARBs to treat hypertension should not be influenced by concerns about COVID19 in the absence of compelling data that ACEs/ARBs either improve or worsen susceptibility to COVID-19 infection nor do they affect the outcomes of those infected

Encourage continuing ACEs/ARBs because there is no evidence linking them to COVID-19 disease severity, and discontinuation of antihypertensive therapy without medica indication could in some circumstances result in harm

Recommend that ACEs/ARBs should not be empirically stopped in patients who are already taking them; in seriously ill patients, changes should be made on a case-by-case basis

Recommend continuing ACEs/ARBs for all patients already prescribed them

Recommend continuing ACEs/ARBs in COVID-19 infection patients due to a lack of evidence to support differential use and the discontinuation of ACEis/ARBs in COVID-19 patients

Strongly recommend continuing ACEs/ARBs until new evidence to the contrary becomes available

Recommend continuing routine use of ACEs/ARBs; patients should not cease BP-lowering medications unless advised to do so by their physician

Recommend that usual antihypertensive therapy is continued given that speculation about risk of ACE inhibitors and ARBs is purely theoretical
March 12, 2020

March 13, 2020

March 13, 2020

March 15, 2020

March 15, 2020

March 16, 2020

March 16, 2020

March 16, 2020

March 17, 2020

March 17, 2020

March 17, 2020

March 18, 2020

March 29, 2020

Used with permission from reference 15 (Sparks MA, Hiremath S, et al.: The coronavirus conundrum: ACE2 and hypertension. NephJC http://www.nephjc.com/news/covidace2). Accessed April 13, 2020.

ACE, angiotensin-converting enzyme; ARB angiotensin receptor blockers,

There is to date no reliable evidence that BP-lowering medications should be routinely dosed at bedtime unless there is a specific evidence-supported indication. The recent Hellenic-Anglo Research into Morning or Night Antihypertensive Drug Delivery
(HARMONY) trial was a prospective randomized crossover trial that tested whether there are differential effects of morning versus evening antihypertensive medication dosing on mean 24-hour ABP levels (36). The HARMONY trial demonstrated that the timing of 
antihypertensive drug dosing (6-11 AM versus 6-11 PM] did not affect mean 24-hour ABP or clinic BP levels in patients with reasonably controlled hypertension on stable therapy with $\geq 1$ antihypertensive medication at baseline. The ongoing Treatment in the Morning versus Evening (TIME) study is comparing the effects of evening versus morning dosing of antihypertensive medication on major CVD outcomes in 21,113 hypertensive patients recruited from population-based settings throughout the United Kingdom and expected to be followed up for 4 years (37). Pending results of the TIME trial (and similar trials when available), it is reasonable to base the timing of antihypertensive medication dosing on patient preference while using as simple a regimen as possible, including long-acting agents (36).

\section{Diuretic Choices}

The majority of the $>103$ million adults in the United States who have a diagnosis of hypertension are candidates for medical antihypertensive therapy. Current guidelines recommend multiple first-line drug classes, including thiazide or thiazide-like diuretics, ACE inhibitors, ARBs, and calcium channel blockers (38). Which of these classes should be preferentially initiated in newly diagnosed hypertensive patients who are free of comorbidities for whom there are compelling treatment indications is uncertain in the absence of strong evidence from randomized clinical trials with head-to-head comparisons. The recently published Large-Scale Evidence Generation and Evaluation Across a Network of Databases for Hypertension (LEGEND-HTN) study used real-world data from nearly 5 million patients with newly diagnosed hypertension in an attempt to answer this question (39). Their main finding was that CVD outcomes, including myocardial infarction, stroke, and hospitalization for heart failure, were reduced in patients treated with thiazide or thiazide-type diuretics compared with other drug classes. The nondihydropyridine calcium channel blockers were inferior to other classes.

Within the thiazide or thiazide-type diuretic class, hydrochlorothiazide is by far the most commonly used agent to treat hypertension. This may relate to the wide availability of multiple doses of hydrochlorothiazide and its desirable safety profile. In contrast, most randomized controlled trials comparing different classes of antihypertensive drugs, including SHEP, ALLHAT and SPRINT, have chosen chlorthalidone as the diuretic because of its demonstrated efficacy for reducing CVD events. In addition, head-to-head comparison studies have shown that chlorthalidone is superior to hydrochlorothiazide in reducing both office BP and 24-hour ambulatory BP $(40,41)$. A recent retrospective observational cohort study, a component of the LEGEND-HTN study cited above, compared hydrochlorothiazide and chlorthalidone with respect to CVD outcomes and safety concerns (42). The study found no differences in CVD outcomes (acute myocardial infarction, hospitalization for heart failure, stroke, and a composite CVD outcome). However, chlorthalidone use was associated with significantly higher risk of several adverse effects, including hypokalemia, hyponatremia, acute renal failure, exacerbation of chronic kidney disease, and type 2 diabetes. The authors concluded that these findings did not support current recommendations to prefer chlorthalidone versus hydrochlorothiazide for hypertension treatment. These conclusions have been questioned because of the brief follow-up times used ( 0.25 years and 0.7 years in separate analyses), time periods too brief to adequately capture CVD benefits of treatment if they were present (43). in the landmark SHEP, ALLHAT, and SPRINT CVD outcome trials, all of which used chlorthalidone as the diuretic, CVD outcome curves started to diverge only after 6 to 12 months. The extremely abbreviated follow-up period in the study of Hripcsak et al. (42) biases toward zero any inherent between-group differences in preventing $\mathrm{CV}$ events. Furthermore, an observational study of a cohort of 12,866 men 35 to 37 years of age, analyzed by Dorsch et al. (44) with a median follow-up time of 6 years, found that chlorthalidone was superior to hydrochlorothiazide in reducing CVD events. Very similar findings were reported in a network meta-analysis of randomized trials by Roush et al. (45).

Clearly, the abbreviated follow-up in the study by Hripcsak et al. (42) puts into question the validity of these retrospective observational data for clinical use. This is particularly important in view of the outstanding safety and efficacy record of chlorthalidone $(43,46)$ and the lack thereof with hydrochlorothiazide (47). Importantly, there are no randomized placebo-controlled trials demonstrating the efficacy of hydrochlorothiazide for reducing CVD events $(45,47)$, and the only head-to-head trial comparing the effects of chlorthalidone with hydrochlorothiazide on CVD outcomes is still in progress in the Veterans Affairs system (48).

For all of these reasons, I strongly prefer chlorthalidone to hydrochlorothiazide for treatment of my hypertensive patients. I limit use of hydrochlorothiazide to situations when single-pill combination therapy that includes a diuretic is desirable for patient convenience, because far more combinations are available with hydrochlorothiazide than with chlorthalidone.

\section{References}

1. Dzau VJ, Balatbat CA: Future of hypertension. Hypertension 74: 450-457, 2019 PubMed

2. Bard DM, Joseph JI, van Helmond N: Cuff-less methods for blood pressure telemonitoring. Front Cardiovasc Med 6: 40, 2019 PubMed

3. Mukherjee R, Ghosh S, Gupta B, Chakravarty T: A universal noninvasive continuous blood pressure measurement system for remote healthcare monitoring. Telemed J E Health 24: 803-810, 2018 PubMed

4. Stein A: A real blood-pressure smartwatch is coming from Omron this year. Available at: https://www.cnet.com/news/omron-heartguide-blood-pressuresmartwatch-ces/. Published Jan 7, 2018. Accessed March 15, 2020

5. Topol EJ: High-performance medicine: the convergence of human and artificial intelligence. Nat Med 25: 44-56, 2019 PubMed

6. Sigmund CD, Carey RM, Appel LJ, Arnett DK, Bosworth HB, Cushman WC, et al.: Report of the National Heart, Lung, and Blood Institute working group on hypertension: barriers to translation. Hypertension 75: 902-917, 2020 PubMed

7. Hoffmann M, Kleine-Weber H, Schroeder S, Krüger N, Herrler T, Erichsen S, et al.: SARS-CoV-2 cell entry depends on ACE2 and TMPRSS2 and is blocked by a clinically proven protease inhibitor. Cell 181: 271-280.e8, 2020 PubMed

8. Vaduganathan M, Vardeny $\mathrm{O}$, Michel T, McMurray J, Pfeffer MA, Solomon SD: Renin-angiotensin-aldosterone system inhibitors in patients with Covid-19. N Engl J Med 382: 1653-1659, 2020 PubMed

9. Wu Z, McGoogan JM: Characteristics of and important lessons from the coronavirus disease 2019 (COVID-19) outbreak in China: summary of a report of 72314 cases from the Chinese Center for Disease Control and Prevention [published online ahead of print Feb 24, 2020]. JAMA doi: 10.1001/jama.2020.2648.PubMed

10. Bavishi C, Maddox TM, Messerli FH: Coronavirus disease 2019 (COVID-19) infection and renin angiotensin system blockers [published online ahead of print Apr 3, 2020]. JAMA Cardiol doi:10.1001/jamacardio.2020.1282. PubMed

11. Ferrario CM, Jessup J, Chappell MC, Averill DB, Brosnihan KB, Tallant $\mathrm{EA}$, et al.: Effect of angiotensin-converting enzyme inhibition and 
angiotensin II receptor blockers on cardiac angiotensin-converting enzyme 2. Circulation 111: 2605-2610, 2005 PubMed

12. Klimas J, Olvedy M, Ochodnicka-Mackovicova K, Kruzliak P, Cacanyiova S, Kristek F, et al.: Perinatally administered losartan augments renal ACE2 expression but not cardiac or renal Mas receptor in spontaneously hypertensive rats. J Cell Mol Med 19: 1965-1974, 2015 PubMed

13. Soro-Paavonen A, Gordin D, Forsblom C, Rosengard-Barlund M, Waden J, Thorn L, et al.; FinnDiane Study Group: Circulating ACE2 activity is increased in patients with type 1 diabetes and vascular complications. J Hypertens 30: 375-383, 2012 PubMed

14. Fang L, Karakiulakis G, Roth M: Are patients with hypertension and diabetes mellitus at increased risk for COVID-19 infection? Lancet Respir Med 8: e21, 2020 PubMed

15. Sparks MA, Hiremath $S$, et al.: The coronavirus conundrum: ACE2 and hypertension. NephJC http://www.nephjc.com/news/covidace2. Accessed March 31, 2020

16. Sparks MA, South A, Welling P, et al.: Sound science before quick judgement regarding RAS blockade in COVID-19 [published online ahead of print Mar 27, 2020]. Clin J Am Soc Nephrol doi: 10.2215/CJN.03530320

17. Zhou F, Yu T, Du R, Fan G, Liu Y, Liul Z, et al.: Clinical course and risk factors for mortality of adult inpatients with COVID-19 in Wuhan, China: a retrospective cohort study. Lancet 395: 1054-1062, 2020 PubMed

18. Burrell LM, Risvanis J, Kubota E, Dean RG, MacDonald PS, Lu S, et al.: Myocardial infarction increases ACE2 expression in rat and humans. Eur Heart J 26: 369-375, discussion 322-324, 2005 PubMed

19. Burchill LJ, Velkoska E, Dean RG, Griggs K, Patel SK, Burrell LM: Combination renin-angiotensin system blockade and angiotensin-converting enzyme 2 in experimental myocardial infarction: implications for future therapeutic directions. Clin Sci (Lond) 123: 649-658, 2012 PubMed

20. Kuba K, Imai Y, Rao S, Gao H, Guo F, Guan B, et al.: A crucial role of angiotensin converting enzyme 2 (ACE2) in SARS coronavirus-induced lung injury. Nat Med 11: 875-879, 2005 PubMed

21. Gu H, Xie Z, Li T, Zhang S, Lai C, Zhu P, et al.: Angiotensin-converting enzyme 2 inhibits lung injury induced by respiratory syncytial virus. Sci Rep 6: 19840, 2016 PubMed

22. Khan A, Benthin C, Zeno B, Albertson TE, Boyd J, Christie JD, et al.: A pilot clinical trial of recombinant human angiotensin-converting enzyme 2 in acute respiratory distress syndrome. Crit Care 21: 234, 2017 PubMed

23. Kreutz R, Algharably EAE, Azizi M, Dobrowolski P, Guzik T, Januszewicz A, et al.: Hypertension, the renin-angiotensin system, and the risk of lower respiratory tract infections and lung injury: implications for COVID-19 [published online ahead of print Apr 15, 2020]. Cardiovasc Res doi: 10.1093/cvr/cvaa097.

24. Salles GF, Reboldi G, Fagard RH, Cardoso CR, Pierdomenico SD, Verdecchia P, et al.; ABC-H Investigators: Prognostic effect of the nocturnal blood pressure fall in hypertensive patients: the ambulatory blood pressure collaboration in patients with hypertension $(\mathrm{ABC}-\mathrm{H})$ meta-analysis. $\mathrm{Hy}$ pertension 67: 693-700, 2016 PubMed

25. Hermida RC: Ambulatory blood pressure monitoring in the prediction of cardiovascular events and effects of chronotherapy: rationale and design of the MAPEC study. Chronobiol Int 24: 749-775, 2007 PubMed

26. Yusuf S, Sleight P, Pogue J, Bosch J, Davies R, Dagenais G; Heart Outcomes Prevention Evaluation Study Investigators: Effects of an angiotensinconverting-enzyme inhibitor, ramipril, on cardiovascular events in highrisk patients. N Engl J Med 342: 145-153, 2000 PubMed

27. Hermida RC, Crespo JJ, Domínguez-Sardiña M, Otero A, Moyá A, Ríos MT, et al.; Hygia Project Investigators: Bedtime hypertension treatment improves cardiovascular risk reduction: the Hygia chronotherapy trial [published online ahead of print Oct 22, 2019]. Eur Heart J doi: 10.1093./ eurheartj/ehz754. 2019 PubMed

28. Mathur P, Kadavath S, Marsh JD, Mehta JL: Chronotherapy for hypertension: improvement in patient outcomes with bedtime administration of antihypertensive drugs [published online ahead of print Nov 14, 2019]. Eur Heart J doi: 10.1093/eurheartj/ehz836.PubMed

29. Kreutz R, Kjeldsen SE, Burnier M, Narkiewicz K, Oparil S, Mancia G: Blood pressure medication should not routinely be used at bedtime We must disregard the data from the HYGIA project. Blood Press 27: 1-2, 2020
30. Carlberg B, Brunstrom M: Invited comment: is bedtime the best time of the day? International Society of Hypertension. Available at: https://ish-world.com/data/uploads/2003-9.pdf. Accessed April 19, 2020

31. Bruno RM, Taddei S: Asleep blood pressure: a target for cardiovascular event reduction? Eur Heart J 39: 4172-4174, 2018 PubMed

32. Pierdomenico SD, Bucci A, Costantini F, Lapenna D, Cuccurullo F, Mezzetti A: Circadian blood pressure changes and myocardial ischemia in hypertensive patients with coronary artery disease. J Am Coll Cardiol 31: 1627-1634, 199 PubMed

33. Kario K, Pickering TG, Matsuo T, Hoshide S, Schwartz JE, Kazuyuki S: Stroke prognosis and abnormal nocturnal blood pressure falls in older hypertensives. Hypertension 38: 852-857, 2001 PubMed

34. Pierdomenico SD, Pierdomenico AM, Coccina F, Lapenna D, Porreca E: Circadian blood pressure changes and cardiovascular risk in elderly-treated hypertensive patients. Hypertens Res 39: 805-811, 2016 PubMed

35. Vrijens B, Vincze G, Kristanto P, Urquhart J, Burnier M: Adherence to prescribed antihypertensive drug treatments: longitudinal study of electronically compiled dosing histories. BMJ 336: 1114-1117, 2008 PubMed

36. Poulter NR, Savopoulos C, Anjum A, Apostolopoulou M, Chapman N, Cross $\mathrm{M}$, et al.: Randomized crossover trial of the impact of morning or evening dosing of antihypertensive agents on 24-hour ambulatory blood pressure. Hypertension 72: 870-873, 2018 PubMed

37. Rorie DA, Flynn RWV, Mackenzie IS, MacDonald TM, Rogers A: The treatment in morning versus evening (TIME) study: analysis of recruitment, follow-up and retention rates post-recruitment. Trials 18: 557, 2017 PubMed

38. Whelton PK, Carey RM, Aronow WS, Casey DE Jr, Collins KJ, Dennison Himmelfarb C, et al.: 2017 ACC/AHA/AAPA/ABC/ACPM/AGS/APhA/ ASH/ASPC/NMA/PCNA guideline for the prevention, detection, evaluation, and management of high blood pressure in adults: a report of the American College of Cardiology/American Heart Association task force on clinical practice guidelines. Hypertension 71: e13-e115, 2018 PubMed

39. Suchard MA, Schuemie MJ, Krumholz HM, You SC, Chen R, Pratt N, et al.: Comprehensive comparative effectiveness and safety of first-line antihypertensive drug classes: a systematic, multinational, large-scale analysis. Lancet 394: 1816-1826, 2019 PubMed

40. Ernst ME, Carter BL, Goerdt CJ, Steffensmeier JJ, Phillips BB, Zimmerman $\mathrm{MB}$, et al.: Comparative antihypertensive effects of hydrochlorothiazide and chlorthalidone on ambulatory and office blood pressure. Hypertension 47: 352-358, 2006 PubMed

41. Dhalla IA, Gomes T, Yao Z, Nagge J, Persaud N, Hellings C, et al.: Chlorthalidone versus hydrochlorothiazide for the treatment of hypertension in older adults: a population-based cohort study. Ann Intern Med 158: 447-455, 2013 PubMed

42. Hripcsak G, Suchard MA, Shea S, Chen R, You SC, Pratt N, et al.: Comparison of cardiovascular and safety outcomes of chlorthalidone vs hydrochlorothiazide to treat hypertension. JAMA Intern Med 180: 542-551, 2020 10.1001/jamainternmed.2019.7454 [Online ahead of print.]PubMed

43. Messerli FH, Roush GC, Oparil S: Comparative efficacy and safety of chlorthalidone (CTD) and hydrochlorothiazide (HCTZ). JAMA Intern Med: 2020

44. Dorsch MP, Gillespie BW, Erickson SR, Bleske BE, Weder AB: Chlorthalidone reduces cardiovascular events compared with hydrochlorothiazide: a retrospective cohort analysis. Hypertension 57: 689-694, 2011 PubMed

45. Roush GC, Holford TR, Guddati AK: Chlorthalidone compared with hydrochlorothiazide in reducing cardiovascular events: systematic review and network meta-analysis. Hypertension 59: 450-1110, 2012 PubMed

46. Oparil S, Cushman WC, Lederle FA: Chlorthalidone versus hydrochlorothiazide in hypertension treatment: do we have the evidence to decide? $\mathrm{Am} J$ Kidney Dis 63: 387-389, 2014 PubMed

47. Messerli FH, Bangalore S: Half a century of hydrochlorothiazide: facts, fads, fiction, and follies. Am J Med 124: 896-899, 2011 PubMed

48. Lederle FA, Cushman WC, Ferguson RE, Brophy MT, Fiore Md LD: Chlorthalidone versus hydrochlorothiazide: a new kind of Veterans Affairs cooperative study. Ann Intern Med 165: 663-664, 2016 PubMed 